\title{
Prevention of the exposure by cyclophosphamide oral tablet
}

\author{
Takae Hanada ${ }^{1,2}$, Yoichiro Takami $^{*}$, Kei Moriyama ${ }^{1}$, Masafumi Oro ${ }^{3}$, Takehiro Ogawa ${ }^{1,3}$, Hiroko Moriyasu ${ }^{3}$, \\ Yuka Inoue ${ }^{1}$, Asako Kanemitsu', Eiko Kawamoto ${ }^{1}$, Ayaka Nagase', Anna Hamahara', Atsuko Yamamoto', \\ Kenichi Shimada ${ }^{1}$, Masashi Takahashi ${ }^{3}$ and Takashi Egawa ${ }^{1}$
}

\begin{abstract}
Background: Unintended exposure to antitumor agents from an oral medicine may place healthcare workers and patients taking medicine at risk. In this study, the exposure to blister pack by CP (cyclophosphamide) and appropriate preventive procedures were examined.

Findings: CP detected inside the blister pack of the tested seven lots by LC-MS/MS ranged from 8.2 to $199.6 \mathrm{ng}$. Raman imaging clearly showed that CP ingredient was completely covered by the tablet coating layer and had not leached out of the tablet. In addition, the amount of CP detected inside the vials was suppressed under the lower detection limit until day 28 , and only $6.0 \mathrm{ng}$ was detected only at day 56.

Conclusions: Various amounts of CP were contaminated to not only the inside of the blister pack but also the outside. This contamination may be caused not only by the manufacturing environment but also by the CP oral tablets themselves through volatilization of CP. Refrigerated storage of CP oral tablets may protect healthcare workers and patients from contact with $\mathrm{CP}$.
\end{abstract}

Keywords: Cyclophosphamide, Oral tablet, Blister pack

\section{Background}

Although antitumor agents are very widely used for the treatment of cancer, they pose a risk to healthcare workers who handle the drugs or work in their vicinity and in patients, even at very low concentration. Exposure by antitumor agents to healthcare workers has been extensively studied over the last two decades. Antitumor agents have been detected in urine, blood and skin from healthcare workers and also in their work environment [1-3]. To prevent the exposure by antitumor agents, country-specific guidelines have been established and closed system devices have been used [4]. However, exposure of healthcare workers to antitumor agents is still observed.

Cyclophosphamide (CP), one of volatile antitumor agents, is also one of the most frequently used drugs. Exposure to $\mathrm{CP}$ in various healthcare working environments

\footnotetext{
* Correspondence: ytakami@shujitsu.ac.jp

'School of Pharmacy, Shujitsu University, 1-6-1 Nishigawara, Naka-ku, Okayama 703-8516, Japan

Full list of author information is available at the end of the article
}

has been found with contamination of working trays, floors, surfaces inside biological safety cabinets, door handles, and injection vials $[5,6]$. CP contamination on the surface of injection vials has been well examined; however, possible contamination on blister packs has been only rarely investigated [7]. The exposure by antitumor agents from oral medicine may place the healthcare workers and patients taking the medicine at risk. Here we evaluated possible exposure to $\mathrm{CP}$ on blister packs and determined ways to prevent $\mathrm{CP}$ exposure.

\section{Materials and methods Chemicals and materials}

$\mathrm{CP}$ oral tablets (Endoxan ${ }^{\circ}$ tablets) were obtained from Shionogi Co. Ltd. Cyclophosphamide monohydrate as a standard CP was obtained from Wako (Osaka, Japan). Hexamethylphosphoramide (HMPA) as an internal standard was obtained from ALDRICH (St Louis, USA). All other chemicals and solvents were of the highest analytical grades available.

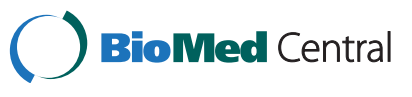




\section{Analytical procedure}

Chromatographic separation was performed using Highperformance liquid chromatography system (Agilent 1100 series; Agilent, USA) and a CAPCELL PAK C18 MGII S-5 $(100 \mathrm{~mm} \times 2.0 \mathrm{~mm}$; i.d., $3 \mu \mathrm{m}$; SHISEIDO, Japan) analytical column at $40{ }^{\circ} \mathrm{C}$. The isocratic mobile phase consisted of mobile phase-A ( $0.1 \%$ formic acid) and mobile phase-B (acetonitrile) $(50: 50, \mathrm{v} / \mathrm{v})$ at a flow rate of $0.2 \mathrm{~mL} / \mathrm{min}$. The solution was filtered using a $0.22 \mu \mathrm{m}$ membrane.

Mass spectrometric detection was performed on an ABSciex API 2000 triple quadrupole mass spectrometer (ABSciex, Toronto, Canada). Data acquisition was performed using the Analyst ${ }^{\mathrm{m} x}$ 1.6.1 software (AB Sciex, Toronto, Canada). The mass spectrometer was operated in the positive ion mode. Optimized instrument settings specific atropine and IS were as follows: curtain gas was $30 \mathrm{psi}$, ion source gas 1 was $70 \mathrm{psi}$, ion source gas 2 was $80 \mathrm{psi}$, ionspray voltage was $4000 \mathrm{~V}$, and turbo heater temperature was $300{ }^{\circ} \mathrm{C}$. Quantification was performed in the multiple reaction monitoring (MRM) mode with mass-to-charge $(\mathrm{m} / \mathrm{z})$ transitions at $261.2>140.1$ for CP and $180.2>135.1$ for IS.

\section{Preparation of the standard solution and quality control samples}

Stock solutions of both CP monohydrate and HMPA were prepared at $1 \mathrm{mg} / \mathrm{mL}$ in methanol. The stock solutions of CP were diluted to $5,10,20,50,100$ and $200 \mathrm{ng} / \mathrm{mL}$ in water containing $0.05 \%$ formic acid and $50 \%$ acetonitrile in water. HMPA stock solutions were diluted and added to both standard and sample solutions at the final concentration of $30 \mathrm{ng} / \mathrm{mL}$.

\section{Sample preparation}

To measure the amounts of $\mathrm{CP}$ attached inside and outside the blister pack separately, the blister pack was washed twice. First, the blister pack containing the tablets was washed by methanol with 10 min of sonication. Next, the CP oral tablets were taken off from the blister pack and residual empty blister pack was washed by methanol with $10 \mathrm{~min}$ sonication. These sample solutions were extracted and measured by LC-MS/MS (Fig. 1).

\section{Raman imaging}

Detailed procedures for Raman imaging are described in Additional file 1.

\section{Results}

The amounts of CP attached to both the inside and outside of each blister pack were separately measured using LC-MS/MS. The CP extraction procedure is described in Fig. 1. As a result, CP ranged from 8.2 to $199.6 \mathrm{ng}$ was detected inside the blister pack for all seven lots (Fig. 2a). In addition, a broad range of CP ( 302.7 ng) was also detected outside the blister pack by some lots (Fig. 2a).

To clarify whether CP had leached out to the outside the tablet, CP localization at a whole tablet or tablet cross section was analyzed using a Raman imaging system. Interestingly, Raman imaging clearly demonstrated that the $\mathrm{CP}$ ingredient was completely covered by the coating layer containing titanium oxide, with no evidence of leaching (Fig. 2b and 2c).

Further, to examine whether the $\mathrm{CP}$ ingredient packed in tablet could volatilize to outside of the tablet and, if present, whether such $\mathrm{CP}$ volatilization could be suppressed by low temperature storage, $5 \mathrm{CP}$ oral tablets were removed from a blister pack and the tablets were stored in a vial at either room temperature (RT) or $4{ }^{\circ} \mathrm{C}$ for $7,14,28$ and 56 days. The average RT was $21.0 \pm 1.7$ $\left({ }^{\circ} \mathrm{C}\right)$ during this experiment (see Additional file 2). Following storage, the $\mathrm{CP}$ attached to the inside of the vials was quantified using LC-MS/MS, respectively. From these analyses, $\mathrm{CP}$ was detected to be $8.0 \mathrm{ng}$ inside a vial stored at RT for 7 days, with the amount of CP detected increasing significantly through day 56 (Table. 1). In addition, the amount of $\mathrm{CP}$ detected inside the vials was suppressed under the lower detection limit until day 28 and only $6.0 \mathrm{ng}$ was detected at day 56 .

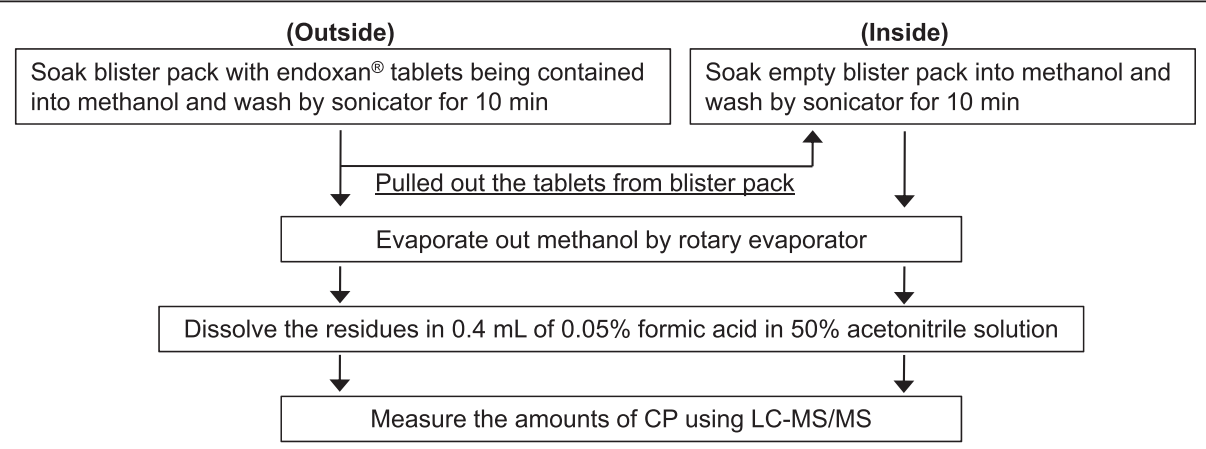

Fig. 1 Schematic representation of sample preparation procedure prior to measurement of cyclophosphamide (CP) attached to the inside or outside of a blister pack separately 
a

\begin{tabular}{|c|c|c|c|c|c|c|c|c|}
\cline { 2 - 10 } \multicolumn{1}{c|}{} & \multicolumn{9}{|c|}{ Amounts of CP detected (ng/piece) } \\
\cline { 2 - 11 } & \multicolumn{3}{|c|}{ Outside } & \multicolumn{5}{c|}{ Inside } \\
\hline Lot. & $\# 1$ & $\# 2$ & $\# 3$ & $\# 4$ & $\# 1$ & $\# 2$ & $\# 3$ & \#4 \\
\hline A & ND & ND & ND & ND & 9.1 & 18.1 & 25.9 & 10.9 \\
\hline B & ND & ND & ND & ND & 29.4 & 39.6 & 68.7 & 25.1 \\
\hline C & ND & $<3$ & ND & ND & 8.2 & 34.8 & 28.0 & 9.3 \\
\hline D & 302.7 & 192.2 & 183.8 & 66.1 & 66.9 & 50.8 & 76.4 & 12.8 \\
\hline E & ND & $<3$ & 32.6 & ND & 47.9 & 132.1 & 199.6 & 37.6 \\
\hline F & ND & $<3$ & ND & ND & 13.2 & 30.8 & 100.2 & 19.2 \\
\hline G & ND & ND & ND & ND & 27.0 & 69.5 & 106.4 & 12.9 \\
\hline
\end{tabular}

b

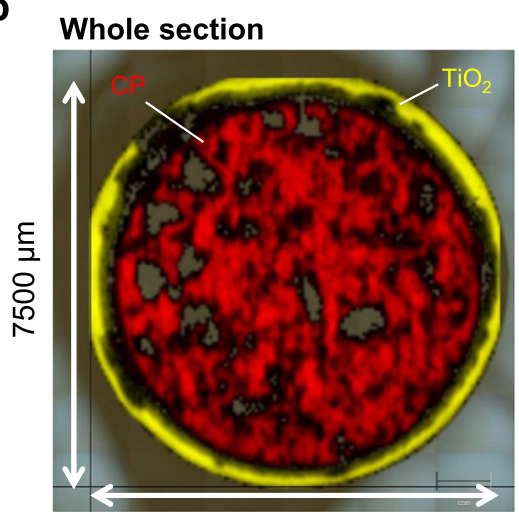

$7500 \mu \mathrm{m}$
C

\section{Partial section}
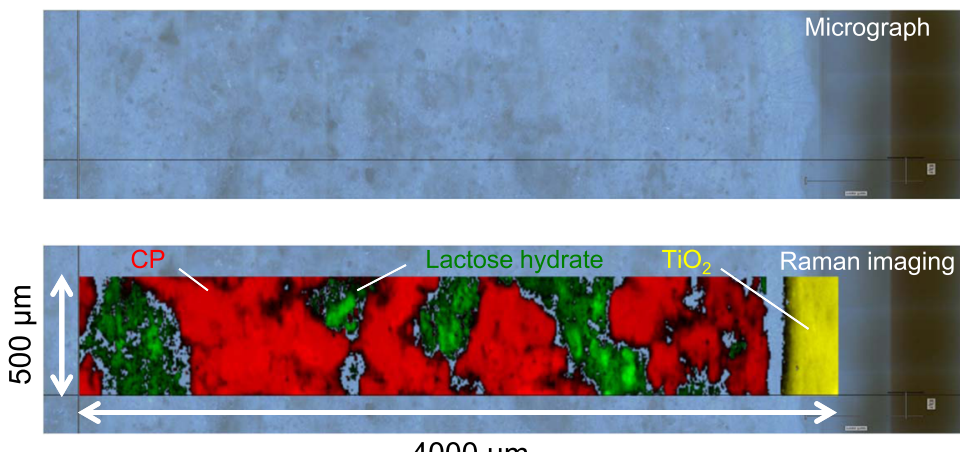

$4000 \mu \mathrm{m}$

Fig. 2 The amount of $C P$ on the inside and outside of the blister packs, and localization of CP within the Endoxan ${ }^{\oplus}$ tablet. a. The amount of $C P$ attached to the inside or outside of the blister pack was measured using liquid chromatography-tandem mass spectrometry (LC-MS/MS). \#1, \#2, \#3 and \#4 are replicate numbers assigned among the same lot. CP, cyclophosphamide; ND, not detected; $<3$, below the detection limit (3 ng/ sheet). $\mathbf{b}$ and $\mathbf{c}$. Raman chemical imaging of (b) CP oral tablet whole and (c) a CP oral tablet cross-section. Red area, CP; Yellow area, titanium oxide; Green area, lactose hydrate

\section{Discussion}

In this study, we demonstrated that various amounts of CP had contaminated both the inside and outside of the blister pack. There are many reports of CP contamination on the surface of $\mathrm{CP}$ injection vials and on their cap covers [7-9]. However, there is currently only one report of CP contamination on both the outside and inside of blister packs. Hedmer et al. reported that $0.5 \mathrm{ng}$ median (range $0.2-3.5 \mathrm{ng}$ ) amounts of $\mathrm{CP}$ was detected on the outside of CP oral tablet blister packs (Sendoxan $50 \mathrm{mg}$ tablets, Sweden) [7]. The authors have pointed out the need for a proper cleaning of manufacturing facilities and equipment to avoid such contamination. As presented in Fig. 2a, the amount of $\mathrm{CP}$ detected outside of the blister

Table 1 Sequential change of CP amounts attached to inside of stocked vials

\begin{tabular}{|c|c|c|c|c|c|c|}
\hline & & & \multicolumn{4}{|c|}{ Stored periods } \\
\hline & & & Day 7 & Day 14 & Day 28 & Day 56 \\
\hline \multirow[t]{4}{*}{ Amount of $C P^{a}$ (ng/vial) } & Vehicle & $R T^{b}$ & ND & ND & ND & ND \\
\hline & & $4{ }^{\circ} \mathrm{C}$ & ND & ND & ND & ND \\
\hline & Endoxan ${ }^{\circledR}$ tablets & $\mathrm{RT}$ & $8.0 \pm 1.2$ & $10.3 \pm 2.7$ & $17.2 \pm 2.0^{*}$ & $49.0 \pm 6.7^{* *}$ \\
\hline & & $4{ }^{\circ} \mathrm{C}$ & ND & $<3$ & $<3$ & $6.0 \pm 1.0^{\# \#}$ \\
\hline
\end{tabular}

a) cyclophosphamide

b) room temperature

Data are represented by mean \pm SD. ${ }^{*} p<0.05 ;{ }^{* *} p<0.01$, Dunnet's multiple comparison test; ${ }^{\# \#} p<0.01$, unpaired $t$-test; ND; not detected, $<3$; under the detection limit (3 ng/vial) 
pack in Lot. D were unaccountably higher than those inside of the blister pack. In this experiment, all lots of blister packs were newly purchased. We ascertained all blister packs uncorrupted. In addition, samples for measurement and $\mathrm{CP}$ standard solutions were prepared on separate table by separate experimenters to avoid artificial contamination of CP. Thus, we think that outside of the blister packs may be randomely contaminated with volatiled $\mathrm{CP}$ from both the manufacturing environment and from the $\mathrm{CP}$ oral tablets themselves. We evaluated the localization of $\mathrm{CP}$ in whole tablet and tablet cross sections using a Raman imaging system to clarify whether $\mathrm{CP}$ had leached out of the tablet. However, tablet's CP ingredient was completely covered by the coating layer and had not leached outside the tablet (Fig. $2 \mathrm{~b}$ and 2c). Thus, $\mathrm{CP}$ was thought to have been volatilized from the tablet.

Furthermore, we showed that such CP contamination through volatilization could be prevented by refrigerated storage (Table. 1). Endoxan ${ }^{\circ}$ tablets are generally recommended for storage at room temperature. Our results showed that $\mathrm{CP}$ contamination inside the blister pack could be suppressed below the detection limit for at least 28 days by refrigeration (Table. 1 ). CP oral tablets are often prepared as a one-dose package in Japan and are not prescribed for more than one month because of efficacy monitoring and the occurrence of adverse effects in treated patients. In fact, the most frequently prescribed period was 28 days from Jan 2015 to Mar 2015 at Muscat Pharmacy (see Additional file 3). Thus, the refrigerated storage of CP oral tablets may protect healthcare workers and patients from unintended contact with the hazardous agents, CP.

\section{Findings}

We demonstrated that various amounts of $\mathrm{CP}$ had contaminated both the inside and the outside of the blister pack. This contamination could be because of both the manufacturing environment and the $\mathrm{CP}$ oral tablets themselves through volatilization. We suggest refrigerated storage of $\mathrm{CP}$ oral tablets to protect healthcare workers and patients from unintended contact CP exposure.

\section{Additional files}

Additional file 1: Supplemental Procedure and Refferences.

Additional file 2: Supplementary Table.

Additional file 3: Supplementary Table.

\section{Abbreviations}

CP: Cyclophosphamide; HMPA: Hexamethylphosphoramide; LC-MS/ MS: Liquid Chromatography-tandem mass spectrometry; HPLC:

High-performance liquid chromatography.

\section{Competing interests}

The authors declare that they have no competing interests.

\section{Authors' contribution}

$\mathrm{TH}$ and $\mathrm{YT}$ : designed all experiments, performed the experiments, analyzed data, and edited the manuscript; KM: analyzed localization of CP by Raman imaging; $\mathrm{MO}, \mathrm{TO}, \mathrm{HM}$ and MT: provide Endoxan ${ }^{\oplus}$ tablet and meaningful discussion; YI, AK, EK, AN, AH and AY: performed the experiments and analyzed data; KS, MT and TE: coordinate this project. All authors read and approved the final manuscript.

\section{Authors' information}

Takae Hanada and Yoichiro Takami are co-first author

\section{Author details}

${ }^{1}$ School of Pharmacy, Shujitsu University, 1-6-1 Nishigawara, Naka-ku, Okayama 703-8516, Japan. ²Department of Pediatrics, Okayama University Hospital, 2-5-1 Shikata-cho, Kita-ku, Okayama 700-8558, Japan. ${ }^{3}$ Muscat Pharmacy, 1290-1 Tamasu, Kita-ku, Okayama 701-1154, Japan.

Received: 25 April 2015 Accepted: 8 June 2015

Published online: 16 July 2015

\section{References}

1. Ensslin AS, Stoll Y, Pethran A, Pfaller A, Rommelt H, Fruhmann G. Biological monitoring of cyclophosphamide and ifosfamide in urine of hospital personnel occupationally exposed to cytostatic drugs. Occup Environ Med. 1994;51:229-33.

2. Nygren $\mathrm{O}$, Lundgren $\mathrm{C}$. Determination of platinum in workroom air and in blood and urine from nursing staff attending patients receiving cisplatin chemotherapy. Int Arch Occup Environ Health. 1997:70:209-14.

3. Fransman W, Kager H, Meijster T, Heederik D, Kromhout H, Portengen L, et al. Leukemia from dermal exposure to cyclophosphamide among nurses in The Netherlands: quantitative assessment of the risk. Ann Occup Hyg. 2014;58:271-82.

4. Sessink PJ, Trahan J, Coyne JW. Reduction in Surface Contamination With Cyclophosphamide in 30 US Hospital Pharmacies Following Implementation of a Closed-System Drug Transfer Device. Hosp Pharm. 2013;48:204-12.

5. Miyake T, Iwamoto T, Tanimura M, Okuda M. Impact of closed-system drug transfer device on exposure of environment and healthcare provider to cyclophosphamide in Japanese hospital. Springerplus. 2013;2:273.

6. Hedmer M, Tinnerberg $H$, Axmon A, Jönsson BA. Environmental and biological monitoring of antineoplastic drugs in four workplaces in a Swedish hospital. Int Arch Occup Environ Health. 2008;81:899-911.

7. Hedmer M, Georgiadi A, Bremberg ER, Jonsson BA, Eksborg S. Surface contamination of cyclophosphamide packaging and surface contamination with antineoplastic drugs in a hospital pharmacy in Sweden. Ann Occup Hyg. 2005;49:629-37

8. Mason HJ, Morton J, Garfitt SJ, Iqbal S, Jones K. Cytotoxic drug contamination on the outside of vials delivered to a hospital pharmacy. Ann Occup Hyg. 2003:47:681-5.

9. Connor TH, Sessink PJ, Harrison BR, Pretty JR, Peters BG, Alfaro RM, et al. Surface contamination of chemotherapy drug vials and evaluation of new vial-cleaning techniques: results of three studies. Am J Health Syst Pharm. 2005;62:475-84.

\section{Submit your next manuscript to BioMed Central and take full advantage of:}

- Convenient online submission

- Thorough peer review

- No space constraints or color figure charges

- Immediate publication on acceptance

- Inclusion in PubMed, CAS, Scopus and Google Scholar

- Research which is freely available for redistribution 